\title{
ASO Author Reflections: Internal Mammary Sentinel Lymph Node Biopsy_Time for the Back of Internal Mammary Staging?
}

\author{
Peng-Fei Qiu, MD and Yong-Sheng Wang, MD, PhD \\ Breast Cancer Center, Shandong Cancer Hospital and Institute, Shandong First Medical University and Shandong \\ Academy of Medical Sciences, Jinan, Shandong, China
}

\section{PAST}

Axillary lymph node (ALN) and internal mammary lymph node (IMLN) are the first-echelon nodal drainage site of breast cancer, yet the primary interest of breast surgeons has been focused on ALN while IMLN were largely ignored, since data from extended radical mastectomy in the 1960s showed no advantages in survival with the IMLN dissection. ${ }^{1}$ With the prominent survival benefit of IMLN radiation, more attention should be paid again to the staging and management of IMLN. Although the internal mammary sentinel lymph node biopsy (IM-SLNB) is a minimally invasive IMLN staging technique, its routine performance remains controversial for the following reasons. $^{2}$ First, the internal mammary sentinel lymph node (IMSLN) were only visualized in a small proportion of patients $(15 \%)$ with traditional radiotracer injection technique, which has been the restriction for IM-SLNB. Second, the IM-SLNB was only performed in cNO patients, which led to the low IMLN metastasis rate $(8-15 \%)$ and little clinical relevance.

ASO Author Reflections is a brief invited commentary on the article, "Internal Mammary Sentinel Lymph Node Biopsy in Clinically Axillary Lymph Node-Positive Breast Cancer: Diagnosis and Implications for Patient Management," Ann Surg Oncol. (2019). https://doi.org/10.1245/s10434-019-07705-0.

(C) The Author(s) 2019

First Received: 4 September 2019; Published Online: 6 November 2019

Y.-S. Wang, MD, PhD

e-mail: wangysh2008@aliyun.com

\section{PRESENT}

One of the primary goal of breast surgery nowadays is nodal staging, which will not be completed without both axillary and IM-SLNB. We tried injecting radiotracer with modified technique (periareolar intraparenchymal, high volume, and ultrasound guidance) in cN0 patients and got a high IMSLN visualization rate of $71.1 \%$, which laid a technical feasibility for further study and clinical application. ${ }^{3}$ In this study, we performed IM-SLNB in $\mathrm{cN}+$ patients and reconfirmed the IMSLN visualization rate. The IMSLN metastasis rate of patients who received initial surgery and neoadjuvant systemic therapy was $39.8 \%$ and $13.3 \%$, respectively. Patients who received IMSLNB will have more accurate nodal staging, which might potentially affect the therapeutic strategies, including individual IMLN irradiation. ${ }^{4}$ As a minimally invasive staging technique, we suggest that IM-SLNB should be routinely performed during mastectomy, especially in $\mathrm{cN}+$ patients, and performed selectively during lumpectomy in high IMLN metastatic risk patients (positive-ALN and/or medial tumor), as an additional 3-cm skin incision might be required. ${ }^{5}$

\section{FUTURE}

High visualization rate and low false-negative rate are prerequisites for the widespread of IM-SLNB. The question arises as to whether IMSLN detected with the modified technique should be considered as the "true" IMSLN. We are conducting two prospective multicenter studies: CBCSG026 trial (NCT03541278) was designed to verify the repeatability of this high IMSLN visualization rate in patients with both ALN negative and positive breast cancer (a minimum of 350 patients for enrollment); the CBCSG027 trial (NCT03024463) of IM-SLNB followed 
by the $1^{\text {st }}$ to 3 rd intercostal IMLN dissection was designed to verify the IM-SLNB accuracy in ALN-positive patients (at least 40 patients with positive IMLN required). Seven centers have enrolled more than three quarters patients in both trials before April 30. The overall IMSLN visualization rate is $68.2 \%$ with IM-SLNB success rate of $94.9 \%$. The overall IMLN positive rate is $42.0 \%$ with a false negative rate of $2.9 \%$ (data not shown). We hope these two trials could provide clinical practice-changing evidence.

DISCLOSURES The authors have no conflicts of interest to disclose.

OPEN ACCESS This article is distributed under the terms of the Creative Commons Attribution 4.0 International License (http://crea tivecommons.org/licenses/by/4.0/), which permits unrestricted use, distribution, and reproduction in any medium, provided you give appropriate credit to the original author(s) and the source, provide a link to the Creative Commons license, and indicate if changes were made.

\section{REFERENCES}

1. Veronesi U, Marubini E, Mariani L, et al. The dissection of internal mammary nodes does not improve the survival of breast cancer patients. 30-year results of a randomised trial. Eur J Cancer. 1999;35:1320-5.

2. Qiu PF, Liu YB, Wang YS. Internal mammary sentinel lymph node biopsy: abandon or persist? Onco Targets Ther. 2016; 9: 3879-82.

3. Qiu PF, Cong BB, Zhao RR, et al. Internal mammary sentinel lymph node biopsy with modified injection technique: high visualization rate and accurate staging. Medicine. 2015;94:e1790.

4. Cong BB, Qiu PF, Wang YS. Internal mammary sentinel lymph node biopsy: minimally invasive staging and tailored internal mammary radiotherapy. Ann Surg Oncol. 2014;21:2119-21.

5. Qiu PF, Zhao RR, Wang W, et al. Internal mammary sentinel lymph node biopsy in clinically axillary lymph node-positive breast cancer: diagnosis and implications for patient management. Ann Surg Oncol. 2019 https://doi.org/10.1245/s10434-019-07705-0.

Publisher's Note Springer Nature remains neutral with regard to jurisdictional claims in published maps and institutional affiliations. 